Abstract for an Invited Paper

for the DPP17 Meeting of

the American Physical Society

\title{
Tamed stability and transport using controlled non-axisymmetric fields in KSTAR $^{1}$
} YONGKYOON IN, Natl Fusion Res Inst

Meticulously orchestrated non-axisymmetric fields enabled KSTAR to explore various paths to tame plasma stability and transport in a very rigorous manner. Given an extremely low level of intrinsic non-axisymmetry, KSTAR has now established high-precision 3-D field control capability that can not only robustly suppress edge localized modes using resonant magnetic perturbation (RMP), but also exclusively alter plasma rotation without invoking particle and energy transport. In highly shaped plasmas (triangularity of $\delta \sim 0.6$ ), we have secured low-n RMP ELM suppressions in a wide range of edge safety factors at $\mathrm{q}_{95}=3.4-6.4$. One of the best $\mathrm{n}=1$ RMP ELM suppressions has been sustained for more than 30 secs (comparable to wall saturation time), satisfying a low edge collisionality $\left(\nu^{*} \sim 0.2\right)$, close to ITER-target. Beyond a typical 3-row RMP configuration, we have newly succeeded in suppressing ELMs with $\mathrm{n}=1$ off-midplane RMPs only, whose configuration is nearly orthogonal to conventional helical RMP structure. Also, a-priori calculation of ideal plasma response led us to identify an optimal window of ELM suppression distinctively separated from mode-lockings. With RMP configuration fixed, a gradual torque control between perpendicular and parallel components helped us access the onset of ELM suppression, strongly supporting a theoretical hypothesis of $\omega_{\perp, \mathrm{e}} \sim 0$ as necessary condition for ELM-suppression, consistent with direct measurement by ECEI. Among non-resonant fields that are quite sensitive to a variation of q95 in NTV physics, a 'mostquiescent' configuration has been identified to fully decouple plasma rotation from particle and energy transport. In support of ITER, KSTAR has conducted a series of experiments regarding RMP misalignment impact and its compatibility with detached plasmas. Preliminary experimental analysis suggests that divertor heat flux might not be sufficiently diffused by misalignment alone, once RMP-driven ELM suppression takes place.

${ }^{1}$ Korean Ministry of Science, ICT and Future Planning 